\title{
How do pre-adolescent children interpret conditionals?
}

\author{
Henry Markovits ${ }^{1} \cdot$ Janie Brisson $^{1} \cdot$ Pier-Luc de Chantal $^{1}$
}

Published online: 28 April 2016

(C) Psychonomic Society, Inc. 2016

\begin{abstract}
Studies examining children's basic understanding of conditionals have led to very different conclusions. On the one hand, conditional inference tasks suggest that young children are able to interpret familiar conditionals in a complex manner. In contrast, truth-table tasks suggest that before adolescence, children have limited (conjunctive) representations of conditionals. We hypothesized that the latter results are due to use of what are essentially arbitrary conditionals. To examine this, we gave a truth-table task using two kinds of conditional rules, Arbitrary and Imaginary categorical rules (If an animal is a bori, then it has red wings) to 9- and 12-yearolds. Results with the Arbitrary rules were consistent with those found in previous studies, with the most frequent interpretation being the Conjunctive one. However, among even the youngest children, the most frequent interpretation of the Imaginary categorical rules was the defective conditional, which is only found with much older adolescents with Arbitrary rules. These results suggest that working memory limitations are not an important developmental factor in how young children interpret conditional rules.
\end{abstract}

Keywords Cognitive development $\cdot$ Mental models $\cdot$ High order cognition

Conditionals are critical components of both linguistic understanding and of logical reasoning. The syntactic and semantic structure of ordinary language conditionals have been

Henry Markovits

henrymarkovits@gmail.com

1 Psychology Department, Université du Québec à Montréal, C.P. 8888, Succ. "A" Montréal, Québec H3C 3P8, Canada extensively studied (e.g., Von Fintel, 2011). Children as young as 3 years of age show sophisticated use of conditional expressions (Scholnick \& Wing, 1992), both as linguistic constructs and as a basis for reasoning. Conditionals are used in a wide variety of contexts, but the most important for our purposes is that they communicate hypothetical relationships that are used as a basis for deductive reasoning. The interface between the linguistic interpretation of conditionals and the way that people reason is thus particularly salient (e.g., Fillenbaum, 1976). Although there are a great many controversies concerning both children's and adults reasoning abilities, one of the more fundamental of these concerns the basic interpretation that children have of conditionals, and consequently, how they can reason with conditionals.

The developmental theory of Barrouillet and colleagues (e.g., Barrouillet \& Lecas, 1999; Gauffroy \& Barrouillet, 2009) predicts that younger children should be restricted to a very limited conjunctive interpretation of basic conditionals. This relies on a mental model analysis of reasoning. Mental model theory (Johnson-Laird \& Byrne, 1991) supposes that reasoners construct internal models of premises using tokenlike representations. Such models are derived from a semantic analysis of combinations of antecedent and consequent terms that are possibly true given the interpretation of the conditional. The full basic interpretation of conditionals of the form "If $\mathrm{P}$ then Q" consists of the three possible combinations of the antecedent and consequent terms that correspond to the true cases of the truth table for conditionals. More specifically, such an interpretation will generate three models corresponding to $<\mathrm{P}$ and $\mathrm{Q}>$, $<$ not-P and $\mathrm{Q}>$ and $<$ not-P and not-Q $>$. With such an interpretation, reasoners will tend to make the logically correct inferences to conditional reasoning problems. However, a key developmental factor is working memory constraints that limit the number of models that can be produced. Barrouillet and Lecas (1999) proposed that 
development of the interpretation of conditionals goes through two preliminary phases before the ability to generate the three models corresponding to the basic conditional is potentially available. The first such phase is found in younger, preadolescent children (12 years or younger) whose working memory is very limited and who are only able to produce the single model corresponding to $<\mathrm{P}$ and $\mathrm{Q}>$.

A child limited to such a model will interpret if-then relations as equivalent to a conjunctive; in other words, they should claim that only the combination of $\mathrm{P}$ and $\mathrm{Q}$ will have a true status, and all other combinations will be false. Older adolescents will be able to produce one additional model, which is <not-P and not-Q>. The two resulting models are equivalent to a biconditional interpretation (if and only if). The full conditional interpretation requiring three models should only be possible for much older adolescents and adults.

Using a variety of tasks intended to directly access the interpretation of conditionals, Barrouillet and colleagues (e.g., Barrouillet, Gauffroy, \& Lecas, 2008; Geoffroy \& Barrouillet, 2009) have indeed found that preadolescent children give primarily conjunctive interpretations, and that more complex interpretations are only found among older adolescents and adults. This hypothesis has important consequences on a variety of levels. It suggests that preadolescent children should be correspondingly constrained in the kinds of conditional inferences that they are capable of making. It also suggests that conditionals should be essentially interpreted as factive (Kiparsky \& Kiparsky, 1971), since any form of suppositional interpretation, such as suggested by Evans and Over (2004) based on adult performance on truth-table tasks would require a more complex representation leaving the possibility of the antecedent term being false.

A very different picture, however, is provided by studies that have examined the ability of preadolescent children to make conditional inferences. Several studies have shown that younger children can make conditional inferences that at least theoretically correspond to interpretations that are much more complex than the simple conjunctive one proposed by Barrouillet and colleagues. For example, children as young as 7 years of age can make complex conditional inferences when reasoning with premises indicating conditional relations between categories and properties., e.g. "If an animal is a dog, then it has four legs" (Markovits, 2000; Markovits \& Thompson, 2008). Importantly, children's ability to do so varies according to the specific content of the conditional rules. For example, reasoning with causal conditionals is relatively difficult, although even with these, preadolescents are able to generate inferences requiring a more complex interpretation (Janveau-Brennan \& Markovits, 1999).

These two sets of results are clearly contradictory. One way of reconciling them is suggested by the importance of content in the developmental pattern of conditional reasoning. Studies suggesting that preadolescents can make conditional inferences corresponding to more complex interpretations have all used conditional rules with interpretable relationships, such as "If category P then property Q" or "If cause P then effect Q." By contrast, the studies used by Barrouillet and colleagues have all used arbitrary conditionals which have concrete terms, but with no explicit or even implicit relationship between the antecedent and the consequent terms, e.g., if something is blue then it is square. This is potentially problematic. Most of the conditionals that are encountered in real life have some interpretable relationship between the terms. In fact, even the more abstract conditionals used in science have interpretable relationships, albeit sometimes with relatively abstract content. Thus, preadolescents might be producing simple conjunctive interpretations of such conditionals simply because they are unable to interpret the relationship between the terms, and not because of some basic working memory limitation. In order to examine this hypothesis, we use a truth table task which has been frequently employed to examine interpretations people make of conditionals.

Truth table tasks present a conditional rule: If $\mathrm{P}$ then $\mathrm{Q}$. Participants are then given examples of the four combinations consisting of true or false forms of the antecedent and consequent terms: P \& Q, not-P \& not-Q, P \& not-Q, not-P \& Q. They are asked to indicate for each one whether it makes the conditional rule true or false, or whether it is irrelevant (see Evans \& Over, 2004 for an overview). A strict conjunctive interpretation of the conditional rule would result in people judging that the $\mathrm{P} \& \mathrm{Q}$ combination will make the conditional rule true, while the other three combinations will make the rule false.

More complex interpretations will generate correspondingly more complex sets of judgments. For example, a biconditional interpretation will result in people judging that the $\mathrm{P} \&$ $\mathrm{Q}$ and the not-P \& not-Q combinations will make the rule true while the other two will make it false. However, it is also the case that people often consider some combinations of antecedent and consequent terms to be irrelevant to a conditional rule, leading to so-called defective truth tables (e.g., Evans \& Over 2004), which can be considered to be incomplete forms of the full interpretation. In fact, the more complex interpretations of arbitrary conditionals found in older adolescents and adults generally correspond to defective versions of biconditional or conditional interpretations.

In order to examine our hypothesis, we repeated a version of the truth-table task with two different forms of if-then rules with preadolescents. We first used the arbitrary conditionals taken from Barrouillet and Lecas's (1999) study. We also examined conditionals with a relational structure corresponding to rules of the form "if category $\mathrm{P}$ then property $\mathrm{Q}$," which are conditionals for which very young children are able to make conditional inferences with concrete content. Ideally, we would use familiar conditionals. However, doing so introduces a potential confound, since it is possible that young 
children might confuse the empirical truth of the statements evaluated in the truth-table task with their effect on the conditional rule. In order to eliminate this possibility, we used completely unfamiliar referents. In order to do so, we told children that these rules concerned imaginary creatures that lived on a strange planet (we refer to these as Imaginary category rules). Thus children were given rules such as "If an animal is a bori, then it has red wings" and asked to evaluate the impact of statements such as "an animal is a malabar and it has red wings" on the truth of the conditional rule. We predicted that conjunctive interpretations would be less frequent with Imaginary category rules than with Arbitrary conditionals.

Finally, it should be noted that more recently Barrouillet and colleagues (Barrouillet, Gauffroy, \& Lecas, 2008; Gauffroy \& Barrouillet, 2009) have extended their theory to heuristic conditionals. We consider the possible implications of this extension when examining the results.

\section{Method}

\section{Participants}

A total of 77 pre-adolescents were examined, of which 35 were in Grade 3 (20 girls, 15 boys; average age: 9 years, 2 months) and 42 were in Grade 6 (21 girls, 21 boys; average age: 12 years, 1 month). All participants were students in an elementary school in the region of Montréal and were native French speakers.

\section{Procedure}

Booklets were distributed to entire classes. Each participant was given a copy of the booklet, with an explanation of what was required given to the entire class. Participants responded to the questions with no further intervention.

Materials Two six-page booklets were prepared. On the first page of each, participants were asked to give their date of birth and gender. They then read the following instructions (translated from the original French):

"Read the following instructions attentively:

In the following pages, you will be presented with several "if-then" rules. Here is one example of this kind of rule:

If it is the nighttime, then it is dark.

For each rule, you will be presented with some statements, one after the other. For each of these, you must decide whether the statement shows that the rule is true, or that the rule is false, or that the statement does not allow showing that the rule is true or false.
You must respond TRUE if the statement shows that the rule is true, FALSE if the statement shows that the rule is false, or ONE CANNOT KNOW, if the statement does not show that the rule is true or false."

Following this, the two if-then rules using arbitrary conditionals were presented, with each rule on the top of a new page. These were:

If a circle is red, then the star is black.

If Melinda wears a red sweater, then she will wear green pants.

Below each rule, four specific cases were presented (corresponding to all combinations of affirming or negating either the antecedent or consequent terms). For each, three choices were given. For example, following the rule "If a circle is red, then the star is black," the following statement, combining a false antecedent and a true consequent, was presented, with the three options directly following it:

1. A circle is green and the star is black.

This statement shows that the rule is:

1. True

2. One cannot know

3. False

The three other statements were:

2. A circle is red and the star is black.

3. A circle is red and the star is white.

4. A circle is blue and the star is green.

The four specific cases corresponding to all combinations of affirming or negating either the antecedent or consequent terms were presented, with the order varied for each rule.

Following this, participants were given the following set of instructions:

"Now, imagine that you have left the earth and that you are travelling in space towards an extraordinary planet. This planet is called Kronus. When you arrive on Kronus, you notice that the animals, the trees and the flowers are all different from those on Earth. In the following pages, you will see some rules and situations that take place on the planet Kronus.

Remember, you must respond TRUE if the statement shows that the rule is true, FALSE if the statement shows that the rule is false, or ONE CANNOT KNOW, if the statement does not show that the rule is true or false."

The next two pages presented the following rules at the top. 
If an animal is a bori, then it has red wings.

If a plant is a mandola, then it has blue leaves.

For each rule, the order of the four statements was randomly varied. It should be noted that, as for the arbitrary rules, the not-P and the not-Q cases were presented as specific examples. In the former this was done with another imaginary category. Glossed translations of the rules are presented in the Appendix.

A second version of this booklet was prepared, with the two categorical relations for imaginary elements presented first, followed by the two arbitrary conditionals.

\section{Results}

We first calculated the distribution of the three responses for each of the four combinations for both the arbitrary and the imaginary category conditional rules (see Table 1). We initially examined responses to the P \& Q and the P \& not-Q combinations. In these cases, understanding of the basic nature of the conditional rules is shown by the true response to the former, and a false response to the latter. We then performed a within subjects ANOVA with numbers of such responses to the two Combinations for both arbitrary and imaginary category rules as dependent variable and Grade as independent variable. This showed only a main effect of Grade, $F(1,74)$ $=3.85, p=.05$. Critically, there was no difference between the arbitrary and imaginary category rules in this respect.

A tendency to give a conjunctive interpretation can then be measured by the degree of false responses to either the not-P \& $\mathrm{Q}$ or the not-P \& not-Q combinations. We performed a second ANOVA with numbers of false responses to these

Table 1 Number of True, False, or Irrelevant responses (out of a total of 2) to the four combinations of antecedent and consequent terms by Rule Type and Grade Level

\begin{tabular}{|c|c|c|c|c|c|}
\hline & \multirow[t]{2}{*}{ Response } & \multicolumn{2}{|l|}{ Grade 3} & \multicolumn{2}{|l|}{ Grade 6} \\
\hline & & Arbitrary & Imaginary & Arbitrary & Imaginary \\
\hline \multirow[t]{3}{*}{$P \& Q$} & True & 1.49 & 1.46 & 1.73 & 1.71 \\
\hline & False & 0.20 & 0.23 & 0.17 & 0.19 \\
\hline & Irrelevant & 0.31 & 0.31 & 0.10 & 0.10 \\
\hline \multirow[t]{3}{*}{ Not-P \& Q } & True & 0.12 & 0.32 & 0.09 & 0.12 \\
\hline & False & 1.09 & 0.71 & 1.62 & 1.14 \\
\hline & Irrelevant & 0.79 & 0.97 & 0.29 & 0.74 \\
\hline \multirow[t]{3}{*}{ Not-P \& not-Q } & True & 0.14 & 0.23 & 0.14 & 0.10 \\
\hline & False & 1.03 & 0.68 & 1.00 & 0.81 \\
\hline & Irrelevant & 0.83 & 1.09 & 0.86 & 1.09 \\
\hline \multirow[t]{3}{*}{ P \& not-Q } & True & 0.20 & 0.09 & 0.12 & 0.17 \\
\hline & False & 1.31 & 1.44 & 1.64 & 1.57 \\
\hline & Irrelevant & 0.49 & 0.47 & 0.24 & 0.26 \\
\hline
\end{tabular}

two Combinations for both arbitrary and imaginary category rules as dependent variable and Grade as independent variable. This showed a significant effect of rule type, $F(1,73)$ $=14.08, \mathrm{p}<.001$, and combination, $\mathrm{F}(1,73)=7.96, \mathrm{p}<.01$, and a significant combination $\mathrm{X}$ grade interaction, $\mathrm{F}(1,73)=$ $4.77, \mathrm{p}<.05$. As predicted, the overall number of false responses was higher for arbitrary rules $(\mathrm{M}=2.39)$ than for imaginary category rules $(\mathrm{M}=1.71)$. More such responses were produced for the not-P \& not-Q combination. There was a decrease in false responses with age, but this was concentrated in the not-P \& not-Q combination.

We then examined patterns of responses based on those identified by Barrouillet and Lecas (1999). We first classified all response patterns for which either not true responses to the P\&Q or not false responses to the P\&not-Q combination were produced, irrespective of any of the responses as Incompatible with a standard interpretation of conditionals. The remaining patterns were classified in the following ways. These were:

Conjunctive This required judging that only the P \& Q statement made the rule true. All other statements were judged to make the rule false.

Defective conditional This required judging that statement corresponding to $\mathrm{P} \& \mathrm{Q}$ made the rule true, while statements corresponding to not-P \& Q and not-P \& not-Q were irrelevant and $\mathrm{P} \&$ not-Q made the rule false.

Defective biconditional This required judging that the statement corresponding to $\mathrm{P} \& \mathrm{Q}$ made the rule true, while not-P \& Q and P \& not-Q make the rule false, while not-P \& not-Q was considered to be irrelevant.

Heuristic These were patterns which used either a matching heuristic (true when the P term was positive, false it was negative) or a single response of either false or irrelevant to all four problems.

Unclassifiable Response patterns with compatible responses to both the P\&Q and the P\&not-Q cases, which did not correspond to previously identified interpretations. Finally, a very small number of responses corresponded to a full biconditional interpretation, so that the total in the table is not quite $100 \%$.

We then determined the overall proportion of these response patterns for the Imaginary Category and Arbitrary rules, for each of the two grade levels (see Table 2). Inspection of Table 2 shows a high percentage of Inconsistent response patterns among the younger children, with a clear decrease in these patterns with age. The most common consistent patterns found with Arbitrary rules corresponded to Conjunctive, followed by Defective Biconditionals. These results are consistent with those obtained by Barrouillet and colleagues, showing that preadolescents 
Table 2 Percentage of response patterns by rule type and grade level (note that biconditional responses are not included here)

\begin{tabular}{lllll}
\hline & Grade 3 & \multicolumn{3}{c}{ Grade 6 } \\
\hline Response patterns & Imaginary & Arbitrary & Imaginary & Arbitrary \\
Conjunctive & 11.4 & 22.6 & 25.0 & 39.3 \\
Defective conditional & 25.7 & 8.6 & 26.2 & 7.1 \\
Defective biconditional & 10.0 & 15.7 & 16.7 & 29.8 \\
Unclassifiable & 7.1 & 8.6 & 3.6 & 1.2 \\
Heuristic & 11.4 & 4.3 & 7.1 & 2.4 \\
Incompatible & 32.9 & 40.0 & 20.2 & 19.0 \\
\hline
\end{tabular}

strongly tend to produce conjunctive interpretations of arbitrary conditionals, with a weaker tendency to produce biconditional interpretations. However, the pattern for Imaginary category conditionals was quite different. Overall, the most common pattern was the Defective Conditional, although among the older children there was an equally high proportion of Conjunctive interpretations. Most critically, the proportion of Defective Conditional interpretations was significantly greater with the Imaginary conditionals than with Arbitrary conditionals both for Grade 3 students, $X^{2}(1, \mathrm{~N}=70)=$ $7.24, p<.01$ and for Grade 6 students, $X^{2}(1, \mathrm{~N}=84)=$ $8.85, p<.01$.

\section{Discussion}

Understanding the nature of young children's interpretations of conditionals is important both within the context of reasoning and for developmental linguistics. The hypothesis proposed by Barrouillet and colleagues, relying on tasks such as the truth table task used here, suggests that younger children simply do not have sufficient working memory capacity to generate interpretations of conditionals that are more complex than the simplest, conjunctive form. By contrast, studies looking at conditional inferences made by children suggest that they have more complex interpretations of conditionals. Our working hypothesis here was that this difference is explicable by the nature of the rules examined. Previous examination of children's interpretation of conditionals have used arbitrary conditionals with concrete content, but without any specification, implicit or explicit, of the nature of the if-then rule. The tendency for children to give conjunctive interpretations might be due to the resulting ambiguity, rather than to some intrinsic cognitive limitation. Our results clearly show this to be the case. When given arbitrary conditionals, the most common consistent interpretations corresponded to the conjunctive pattern, followed by defective biconditionals. By contrast, when given premises using Imaginary category rules using unfamiliar content, but with a clearly stated category to property structure, the most commonly observed consistent pattern was the defective conditional, which was produced at a significantly higher rate than with Arbitrary conditionals. In fact, given the arbitrary nature of the content used in the former, we would argue that these results understate children's ability to make complex interpretations of conditional rules.

One alternative explanation is that children are giving a form of heuristic interpretation of the imaginary category rules. There is evidence that even young children can use pragmatic factors to interpret conditional rules, for example using simple permission schemas (Girotto, Light, \& Colbourn, 1988). Barrouillet and colleagues (Gauffroy \& Barrouillet, 2009) have claimed that processing pragmatic conditionals can be done by a form of heuristic processing which furnishes an interpretation that requires minimal working memory capacity. For example, conditional promises generate pragmatic interpretations equivalent to biconditionals, in which only cases corresponding to $\mathrm{P}$ and $\mathrm{Q}$ and not-P and not$\mathrm{Q}$ make the rule true, while all other combinations make the rule false (Fillenbaum, 1976). Interestingly, they use this distinction to explain why older adolescents and adults often generate defective interpretations of conditionals. The idea is that interpretations resulting from heuristic processing are encoded as corresponding to true cases, while interpretations resulting from a more cognitively costly process requiring active consideration of the nature of the conditional rule ("fleshing out" in mental model terminology) are generated as possibilities, but encoded as truth-functionally irrelevant. If some form of heuristic processing were indeed responsible for the high levels of more complex interpretations observed here, then we would expect that children would be producing complete biconditionals or conditionals when evaluating the Imaginary categorical rules. However, the high levels of defective conditionals produced with the latter is simply not consistent with this interpretation.

One other possibility is that children were somehow systematically misinterpreting the Imaginary rules, due to some unspecified failure to understand conditionals. If this was the case, then we would expect these children to have comparatively more problems with the arbitrary conditionals. We thus compared the percentage of defective conditional interpretations and the percentage of inconsistent interpretations (combining unclassifiable, heuristic, and incompatible patterns) between children who had at least one defective conditional interpretation of the Imaginary rules and those who did not. The former had higher percentages of defective conditional interpretations of arbitrary rules (18.8\% vs. 2.9 $\%)$ and lower percentages of inconsistent interpretations (10.4\% vs. $48.6 \%)$. Thus, children who produced defective conditional interpretations of Imaginary rules actually showed a greater tendency towards consistency and more advanced interpretations of arbitrary conditionals than those who did not do so. 
Thus, these results clearly show that pre-adolescents have sufficient working memory capacity to generate complex representations of basic if-then rules, and invalidate the hypothesized developmental sequence that depends on just this factor. This is in turn consistent with previous cited results showing that young children can make complex logical inferences with appropriate rules and content (Markovits, 2000). How then can one account for the consistently conjunctive interpretations found using arbitrary conditionals? One possible explanation is that these conditionals are interpreted as essentially meaningless (e.g., abstract) by younger children, given the absence of any cues as to the semantics of the if-then rule. Inferential performance with abstract conditionals is very difficult (Markovits \& Vachon, 1990) and shows a developmental pattern quite similar to that observed by Barrouillet and Lecas (1999).

\section{Appendix}

Glossed translations of the conditional rules

Si le cercle est rouge, alors l'étoile est noire.

If the circle is red, then the star is black.

'If the circle is red then the star is black.'

$\mathrm{Si}$ on porte un chandail rouge, alors on met un pantalon vert.

If one wears a sweater red, then one puts on a pant green.

'If one wears a red sweater, then one puts on green pants'

Si un animal est un bori, alors il a des ailes rouges.

If an animal is a bori, then it has wings red.

'If an animal is a bori, then it has red wings.'

Si une plante est une mandola, alors elle a des feuilles bleues.

If a plant is a mandola, then it has leaves blue.

'If a plant is a mandola, then it has blue leaves.'

\section{References}

Barrouillet, P., Gauffroy, C., \& Lecas, J.-F. (2008). Mental models and the suppositional account of conditionals. Psychological Review, $115(3), 760-771$.

Barrouillet, P., \& Lecas, J.-F. (1999). Mental models in conditional reasoning and working memory. Thinking \& Reasoning, 5(4), 289-302.

Evans, J. S. B. T., \& Over, D. E. (2004). If. New York, NY: Oxford University Press.

Fintel, K. von (2011). Conditionals. In K. von Heusinger, C. Melenborn, P. Portner (Eds). Handbücher Zur Sprach-Und Kommunikationswissenschaft / Handbooks of Linguistics and Communication Science, De Gruyter, p. 1515-1538. Doi: 10.1515/ 9783110255072.1515

Janveau-Brennan, G., \& Markovits, H. (1999). The development of reasoning with causal conditionals. Developmental Psychology, 35(4), 904-911.

Johnson-Laird, P. N., \& Byrne, R. M. J. (1991). Deduction. Hillsdale, NJ: Laurence Erlbaum Assoc.

Fillenbaum, S. (1976). Inducements: On the phrasing and logic of conditional promises, threats, and warnings. Psychological Research, 38, 231-250.

Gauffroy, C., \& Barrouillet, P. (2009). Heuristic and analytic processes in mental models for conditional: An integrative developmental theory. Developmental Review, 29, 249-282.

Girotto, V., Light, P., \& Colbourn, C. (1988). Quarterly Journal of Experimental Psychology, 40(3), 469-482.

Kiparsky, P., \& Kiparsky, C. (1971). Fact. In D. D. Steinberg \& L. A. Jakobovits (Eds.), Semantics. An Interdisciplinary Reader in Philosophy, Linguistics and Psychology (pp. 345-369). Cambridge: Cambridge University Press.

Markovits, H. (2000). A mental model analysis of young children's conditional reasoning with meaningful premises. Thinking \& Reasoning, 6(4), 335-347.

Markovits, H., \& Vachon, R. (1990). Conditional reasoning, representation, and level of abstraction. Developmental Psychology, 26(6), 942-951.

Markovits, H., \& Thompson, V. (2008). Different developmental patterns of simple deductive and probabilistic inferential reasoning. Memory and Cognition, 36(6), 1066-1078.

Scholnick, E. K., \& Wing, C. S. (1992). Speaking deductively: Using conversation to trace the origins of conditional thought in children. Merrill-Palmer Quarterly, 38(1), 1-20. 